\title{
Management im Spital neu erfinden? (Teil 2)
}

\author{
Christof Schmitz, \\ Peter Berchtold
}

Korrespondenz:

Dr. Christof Schmitz

College-M

Freiburgstrasse 41

CH-3010 Bern

christof.schmitz@college-m.ch

www.college-m.ch
Im ersten Teil dieser Artikelserie [1] haben wir aufgezeigt, dass gelingendes Führen in Spitalorganisationen kaum mit Standardmanagementtechniken zu haben ist. Bekannte und weitum praktizierte Managementtechniken wie Strategisches Management oder Qualitätsmanagement drohen «korrumpiert» zu werden, wenn sie unbesehen angewandt werden. Spitäler sind anders (als andere Organisationen), und die Frage ist damit offen, was diese Andersartigkeit für das Management von Spitälern bedeuten soll. In diesem Beitrag möchten wir dieser Frage nachgehen.

\section{Sparten und Kreisel}

Fungierten Spitäler lange Zeit als eine Art Nebeneinander von berufsständisch orientierten Sparten - Medizin, Pflege, Verwaltung -, sehen wir heute vielfache Bewegung in Richtung mehr Integration. Grosse Spitäler bemühen sich z. B. um die Formung von Bereichen oder Departementen, um der de facto unmöglichen Führungsspanne etwa von bis zu 40 Kliniken auf Universitätsspitalniveau Herr zu werden und der zunehmenden Vernetzung medizinischer Leistungen Rechnung tragen zu können. Wie verschiedene Beispiele im In- wie Ausland zeigen, gelingt es dabei nur begrenzt, klare Unterstellungsverhältnisse zu generieren. Was sich abzeichnet, entspricht vielmehr dem Bild des berühmt-berüchtigten Verkehrskreisels «Magic Roundabout» von Swindon/UK (Abb. 1). Dieser Kreisel, der einzigartigerweise in sich nochmals 5 Kreisel birgt, garantiert trotz seiner beträchtlichen Komplexität einen sichereren Verkehrsfluss als eine (zu) einfache Rotlichtanlage [2]. Allerdings ist er wesentlich dann «erfolgreich», wenn seine Benutzer bereits Erfahrung mit ihm sammeln konnten. Unerfahrene Autofahrer drohen gelegentlich, Schweissausbrüche produzierend, in den fünf Kreiseln verloren zu gehen. Dafür kann man dann in Swindon das offizielle «I survived the Magic Roundabout»T-Shirt erwerben.

Wird es einmal ein T-Shirt für CEOs und andere Manager «I survived the Hospital» geben? Diese scherzhafte Frage bezieht sich auf eine reell existierende Problemstellung, nämlich die Schwierigkeit, eine Mehrzahl disziplinär und via Berufsgruppeninteressen differenzierte Leistungsbündel miteinander zu integrieren. Beispielhaft drückt sich das in der Formierung von sogenannten Klinik- oder Departementmanagern aus oder in der gelegentlich etablierten Unterstellung der Pflege unter die Klinikdirektoren. Beides bedeutet ein Abgehen von der alten Spartenorganisation. Beides sind Versuche, den tatsächlichen Aufwänden und Leistungsgegebenheiten Rechnung zu tragen. Klinik- und Departementmanager als Anerkenntnis der höheren Aufwände für betriebswirtschaftliche, administrative und koordinative Funktionen und die Neuorganisation der Pflege als Anspruch, medizinische Prozesse und Führungsstrukturen stärker miteinander in Einklang zu bringen. Gerade das Zweite zeigt aber auch die Problematik, um die es geht.

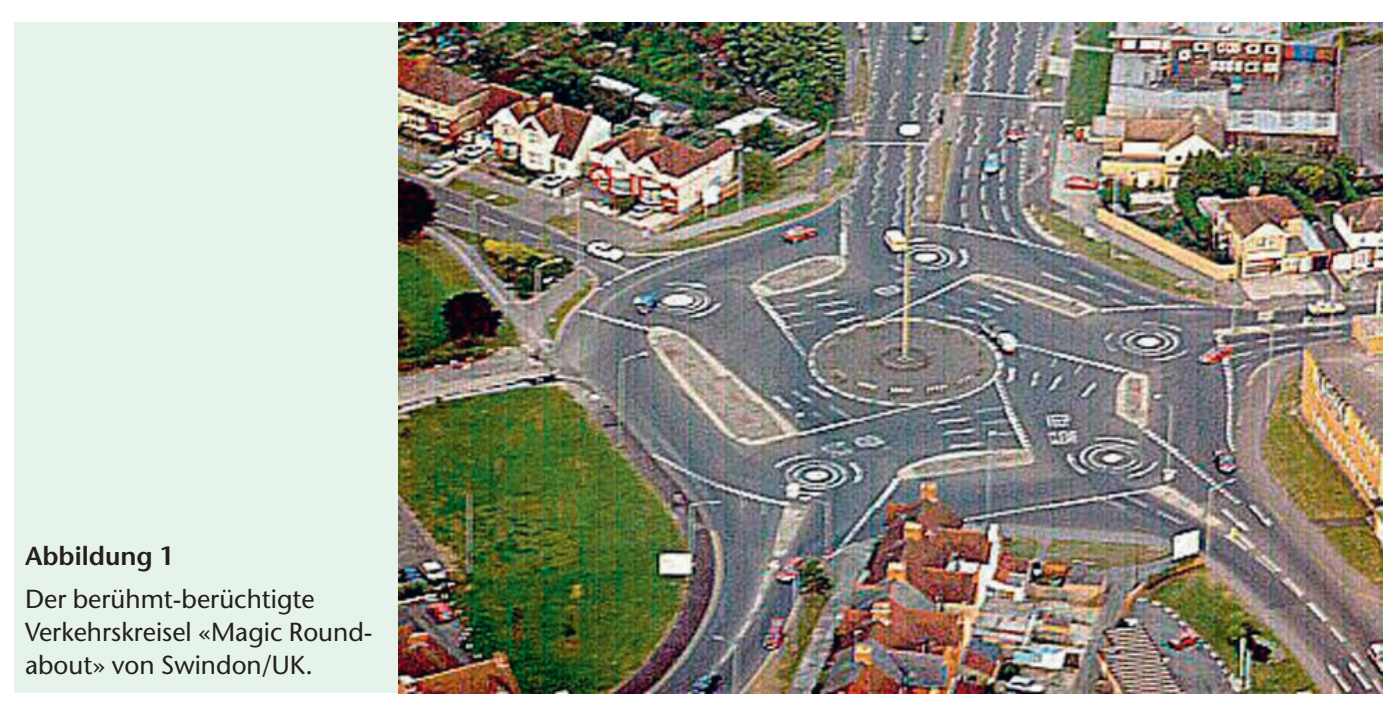


Zwar ist Pflege nun unterstellt, aber fachlich weitgehend autonom und diesbezüglich der jeweiligen Pflegedirektion unterstellt. Exakt diese Kombinationen von Unterstellung und (relativer) Autonomie machen Spitalsorganisationen so komplex und erinnern an den «Magic Roundabout». Es geht um ein Vernetzen von Leistungen bzw. Leistungsströmen, das mehr ist ein Aneinanderfügen serieller Aufgaben.

\section{Vernetzen und Führen}

Bei Verkehrskreiseln steht die Aufrechterhaltung von Verkehrflüssen im Vordergrund (vs. Stoppen von Flüssen durch Ampelanlagen). Wenn wir dieses Bild auf Organisationen übersetzen wollen, sprechen wir von Netzwerken, d.h. lose mit einander gekoppelten, relativ autonomen Einheiten, die miteinander in ihrer Leistungserbringung zu integrieren sind. Es darf erwartet werden, dass solche Arten von Organisationen eigene Führungsformen benötigen. Man kann sich das leicht vor Augen führen, wenn man sich vorstellt, was passiert, wenn in einem sich weitgehend selbst regulierenden Netzwerk jemand versucht, stark strukturierend einzugreifen. Das hat nur begrenzte Erfolgschancen. In einem eigenen Forschungsprojekt «Managing Professionals» des College-M und der Universität Basel gingen wir dem Führungsverständnis von Spitalmanagern und Chefärzten nach [3]. Auffallend und der obigen Vermutung tatsächlich entsprechend war, wie stark die Spitalmanager Beziehungsgestaltung in den Vordergrund rücken. Sie benötigen funktionierende Beziehungen, um ihren eigenen Anliegen Gewicht verschaffen zu können und Mediziner wie Pflegende in die Wahrnehmung übergeordneter Belange integrieren zu können. Die im Vergleich zu anderen Führungskontexten starke Gewichtung der «Beziehungsarbeit» und des «Integrierens» ist bemerkenswert. Auch wenn einem CEO eines Industrieunternehmens Beziehungen zu seinen Untergebenen nicht gleichgültig sein werden, wird er sie im Vergleich mit den Spitalmanagern als weniger vordringlich ansehen. Das erscheint plausibel angesichts des unterschiedlichen Kontextes im Spital. Im Netzwerk der Kliniken und Institute braucht es funktionierende Beziehungen auf der Basis von Wertschätzung und Vertrauen, will man zu gemeinsamen Zielen kommen - Beziehungsgestaltung wird zentral.

Spitalmanager artikulierten eine Ambivalenz zu Themen wie «Anpacken, bewegen und verändern» oder «sich behaupten, sich durchsetzen». Beides sind Themen eines eher instruierenden Stiles, der Einfluss von «Amtes wegen» oder durch formale Macht geltend machen kann. Für
Spitalmanager kann eine solche Strategie riskant sein: Sie deuten in den Interviews an, dass man formal gesehen Entscheidungskompetenz hätte, sie aber ungern einsetzen möchte, weil konfrontative Durchsetzung Beziehungen gefährden kann. Gleichwohl stehen die Spitalmanager immer wieder unter dem Druck, zum Beispiel bei politischen Vorgaben von aussen, sich dieser Variante des Durchsetzens zu bedienen. Ein Verzicht auf diese Variante ergibt dort Sinn, wo Commitment von zentraler Bedeutung ist und es wesentlich darum geht, die Zustimmung wichtiger Akteuren zu gewinnen, und diese Akteure über ausgeprägte Autonomiepotentiale verfügen. Die Ambivalenz zur Durchsetzung liegt darin, dass sie die Gefahr birgt, konflikthafte Zustände heraufzubeschwören, in denen die Mediziner Entscheidungen weder mittragen noch umsetzen (mit den entsprechenden Vertrauensverlusten). Die Gefahr auf der anderen Seite kann aber auch sein, dass zu wenig Durchsetzung stattfindet, indem wichtige Fragen in ewigen Aushandlungsprozessen stecken bleiben und ihrerseits suboptimale Lösungen Platz greifen. Spitalmanagement scheint damit der Fahrt zwischen Scylla und Charybdis zu gleichen: eskalierende Konflikte auf der einen und einschläfernde Kompromisse auf der anderen Seite.

\section{Komplexität}

Die Komplexität der Vernetzungsarbeit in Spitäler ergibt sich nicht nur aus der Unterschiedlichkeit der Leistungen und der Differenzierung der Berufsgruppen oder Professionen, sondern nicht zuletzt auch aus den verschiedenen Führungsformen der einzelnen Gruppen. Insbesondere in Hinblick auf die Ärzteschaft wird das verstärkt diskutiert, indem kritisch befragt wird, ob denn Klinikdirektoren die Vielzahl ihrer Agenden noch hinreichend bewältigen können und ob Führungsarbeit nicht zu kurz komme. Tatsächlich gibt hier einiges zu diskutieren, zugleich aber ist zur Kenntnis zu nehmen, dass die Kombination von Führungs- und Facharbeit, wie sie in der ärztlichen Profession vorherrscht, den Hinweis auf eine spezielle Art von Geschäft bedeutet.

Wir finden diese Kombination des «producing - managing - leading», also die Kombination von Fach- und Führungsarbeit, auch in anderen Organisationen, insbesondere den sogenannten professionellen Organisationen, etwa Beratungsfirmen, Rechtsanwaltskanzleien, Investmentbanken u. Ä. Professionelle Organisationen sind solche, die hochprofessionelle, spezifisch auf jeweilige Kundenprobleme zugeschnittene Dienstleistungen oder Praktiken offerieren. Sie charakterisieren sich im Kern dadurch, dass sie 
(anstelle von standardisierten Produkten) spezifisches professionelles (z. B. medizinisches) Knowhow sowie dessen Beurteilung und Anwendung verkaufen. Organisationen dieses Typs unterscheiden sich markant von produktproduzierenden Firmen. Letztere sind im Kern funktional, z. B. in Beschaffung, Marketing, Produktion, Vertrieb, gegliedert, professionelle Organisationen disziplinär (z. B. im Spital in medizinische Fachkliniken, Pflegeeinheiten). Das hat Konsequenzen für Management und Führung, denn die in produktproduzierenden Firmen übliche Trennung zwischen Führung und Produktion (ein CEO ist kaum gleichzeitig Führender und funktionaler Spezialist) ist in professionellen Organisationen aufgehoben, indem ein Chefarzt beispielsweise (fast) immer als Klinikchef und Arzt tätig ist. Diese Integration ist folgenreich für Führungsmodelle und -verständnisse. Dieses Unterschiedlich-Sein ist gerade für ungeübte Manager verwirrend und letztlich wohl Ursache für viele fehlgeschlagene Managementinitiativen (s. «Korruption») sowohl in weiteren professionellen Organisationen als auch in Spitälern [4].

\section{Führen in komplexen Kontexten}

Wenn Führen und Macht infolge netzwerkartiger Verhältnisse diffus sind, hängt Führungserfolg wesentlich an der Fähigkeit, starke und dauerhafte (Beziehung-)Netzwerke aufzubauen und in diesen Einfluss zur Geltung zu bringen [5]. Netzwerkfähigkeit erfordert gleichzeitiges Verfolgen von Inhalten in Form von Projekten und Zielen und der Beziehungen im Netzwerk. Während es beim Ersteren bereits oftmals um komplexe sachliche Thematiken geht, erhöht Zweiteres, die Beeinflussung wichtiger Akteure und vor allem die Übersetzung von Themen in netzwerkrelevante (professionelle) Bedeutungen, nochmals wesentlich die Komplexität des Geschehens.

Kommen (produktorientierte) Managementexperten hinzu, liegt es nahe, Vereinfachungen und Standardprozeduren einzurichten. Der an sich sinnvolle Wunsch, es so einfach wie möglich zu halten, führt dazu, dass durch eine ausgeprägte «Administrierung» (Formalisierung) Probleme zu bewältigen gesucht werden, die so nicht bewältigt werden können - diese sogenannte «Administrationsfalle» ist allgegenwärtig in Spitälern. Versucht man komplexe Situationen (z. B. die Organisation eines Universitätsspitals) mit (zu) einfachen Methoden anzugehen, droht man in die Nähe von (hoffnungslos vertrackten) For- men, wie sie in Abbildung 2 symbolisch dargestellt sind, zu gelangen.

In einem dritten Teil dieser Beitragsserie wollen wir aufzeigen, was das konkret für Prozesse des Führens in Spitälern heisst.

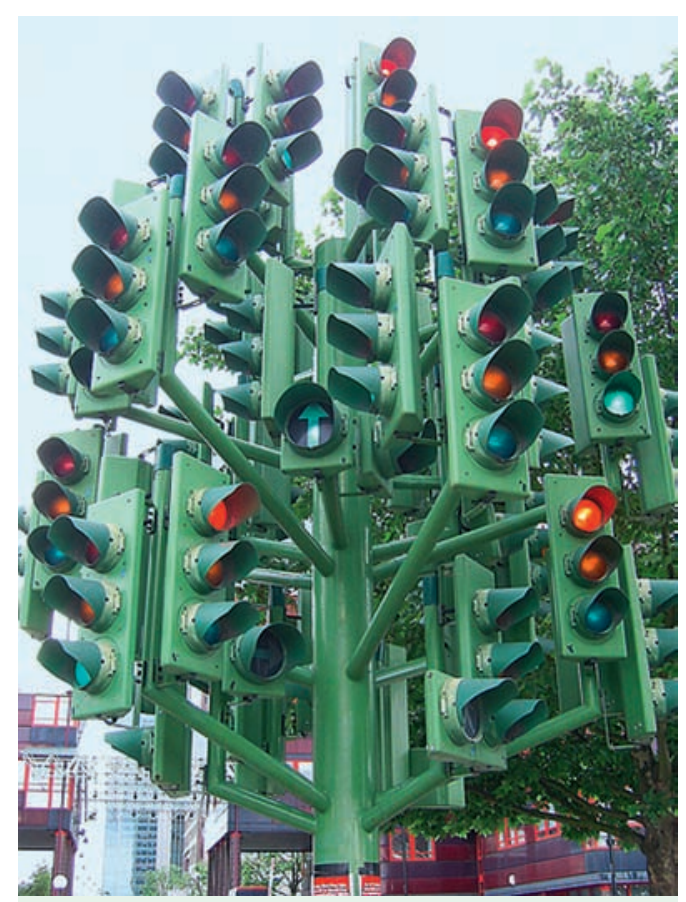

Abbildung 2

Komplexe Strukturen mit (zu) einfachen Methoden anzugehen kann zu (hoffnungslos vertrackten) Formen führen.

\section{Literatur}

1 Schmitz C, Berchtold P. Management im Spital neu erfinden? (Teil 1). Schweiz Ärztzeitung. 2008;89(30/31):1306-8.

2 Nambisan SS, Parimi V. A comparative evaluation of the safety performance of roundabouts and traditional intersection controls. Institute of Transportation Engineers. ITE Journal, March 2007. www.ite.org/itejournal/index.asp.

3 Berchtold P, Endrissat N, Müller WR, Schmitz C. Managing Professionals - Führung in Spitälern. Projektbericht. www.college-m.ch/uploads/ media/Managing_Professionals_Executive Summary_02.pdf.

4 Delong TJ, Gabarro JJ, Lees RJ. Professional service firms - a breed apart. In: Delong TJ, Gabarro JJ, Lees RJ. When Professionals Have to Lead - a New Model for High Performance. Harvard: Harvard Business School Press; 2007.

5 Denis J-L, Lagley A, Rouleau L. Rethinking Leadership in Public Organizations. Oxford: Oxford Handbook of Public Management; 2005. p. 446-67. 